\title{
Alternativa ecológica y económica para la obtención de postura de Coffea arabica L.
}

\author{
Ecological and economical alternative for Coffea arabica L. seedling obtainment
}

\section{Luis Gustavo Moisés M. ${ }^{1}$; Yonger Tamayo A. ${ }^{2}$; Fernando Vicente Barraza A. ${ }^{3}$}

1 Profesor e investigador, M.Sc, Universidad de Guantánamo, Guantánamo, Cuba, moises@cug.co.cu

2 Profesor e investigador, M.Sc, Universidad de Guantánamo, Guantánamo, Cuba, yongertamayo@cug.co.cu

3 Profesor e investigador, I.A., Ph.D. Universidad de Córdoba, Córdoba, Colombia, fbarraza@correo.unicordoba.edu.co

Fecha recepción: Marzo 27 de 2015

Fecha de aceptación: Mayo 04 de 2015

\section{RESUMEN}

El experimento se realizó en la finca Limonar situada en el municipio El Salvador, con el objetivo de evaluar el comportamiento de las posturas de café (Coffea arabica L.) con dos cepas de hongos micorrízicos arbusculares (HMA) en el sistema productivo cafetalero de Limonar. Se empleó el sistema de siembra en viveros en bolsa de polietileno con un total de 360 plantas, de ellas 120 se tomaron para ser evaluadas. Se utilizó un diseño completamente aleatorizado con tres tratamientos y cuatros réplicas, resultantes de la inoculación de las semillas de cafeto con dos cepas de HMA (Glomus cubense y Rhizophagus intraradices) y un control sin inoculación, el mismo se montó sobre un sustrato conformado por una relación suelo materia orgánica (Vacuno) 5:1. Se evaluaron variables morfológicas como pares de hojas, área foliar $\left(\mathrm{cm}^{2}\right)$, altura de las plantas, largo de la raíz, materia seca (g) y el Índice de eficiencia (IE). Se realizó un análisis de varianza de clasificación simple, los resultados arrojaron que la inoculación de los hongos micorrízicos arbusculares incrementó todos los indicadores estudiados para las posturas de café en las condiciones de estudio, presentando beneficios ambientales y económicos.

Palabras clave: café, Coffea arabica L., hongos micorrizogenos arbusculares (HMA), materia orgánica, inoculación. 


\begin{abstract}
The experiment was made in the Limonar located in the El Salvador municipality, with the objective to evaluate the behavior of the coffee seedling Coffea arabica L. with two stocks of arbuscular mycorrizal fungi (HMA) in the coffee productive system of Limonar. The system of sowing in breeding grounds in polyethylene stock market was used with a total of 360 plants, from them 120 were taken to be evaluated. It was Completely used a design randomized with three treatments and retorts four, resultants of the inoculation of the seeds of coffee plant with two stump of HMA Rhizophagus intraradices and Glomus cubense and a control without inoculation, the same one mounted on a substrate conformed by a relation soil organic matter (Bovine) 5:1. Morphologic variables like pairs of leaves, area to foliar $\left(\mathrm{cm}^{2}\right)$, height of the plants, length by the root, dry matter (g) and the Index of efficiency were evaluated (IE). An analysis of variance of simple classification was made, the results threw that the inoculation of the arbuscular mycorrizal fungi increased all the indicators studied for the coffee seedling in the conditions of study, presenting environmental benefits and ecological.
\end{abstract}

Keyword: coffee seedling, arbuscular mycorrhizal fungi, organic matter, inoculation.

\section{INTRODUCCIÓN}

El reconocimiento, por parte de la comunidad mundial, de la importancia de los bienes y servicios que brindan los cafetos es cada día mayor; por la protección y conservación de los recursos naturales como los suelos, las aguas, las zonas costeras, los recursos de la biodiversidad, el equilibrio y mejoramiento del medio ambiente en general, son funciones insustituibles de los ecosistemas cafetaleros (Sánchez et al., 2003).

En el programa Plan Turquino para el incremento de la producción de café del Grupo Empresarial de Agricultura de Montaña se proyecta, para el período 2010 - 2015, la siembra de 6088,7 ha para el cultivo de café que representan el $30 \%$ de las áreas a sembrar en todo el país (Bustamante et al., 2010).

Por su parte, las necesidades nutricionales para las plántulas o posturas de cafetos en Cuba se garantizaban tradicionalmente con abonos orgánicos (Sánchez et al., 2003). No obstante, a estas alternativas, se ha logrado en la actualidad la inserción de los hongos micorrízicos arbusculares con el propósito de incrementar la producción de café in situ (Sánchez et al., 2006).

En éste orden, los hongos micorrízicos arbusculares (HMA) son microorganismos que permiten mejorar el desarrollo de los cultivos, cuando interactúan con las plantas, creando simbiosis entre sí. También están presentes en cerca del 95\% de los cultivos agrícolas y pueden aumentar los procesos de absorción y traslocación de nutrientes en las plantas (Rivera et al., 2007).

En las cercanías de la raíz, los filamentos hifales son capaces de explorar volúmenes de suelo mucho mayores que las raíces no micorrizadas, estimulando de esta forma el crecimiento, reflejado en un aumento de la producción de masa aérea y radical. También pueden mejorar el aprovechamiento de los fertilizantes minerales y los nutrientes del suelo (Sánchez, 2001). 
En este sentido, los hongos micorrízicos abusculares en la producción agrícola han sido empleados en diferentes cultivos de interés económico para la agricultura, siendo posiblemente la producción de posturas de cafeto, una de las áreas en la cual, desde hace años, más resultados con repercusión práctica hayan sido encontrados (Sánchez et al., 2009).

Rivera et al. (2007) plantean que el efecto de la simbiosis micorrízica en los agroecosistemas de especies perennes (café), permitirá extender a los mismos los efectos beneficiosos de la simbiosis micorrízica relacionados con los incrementos en la absorción de los nutrientes y agua y por tanto mayor capacidad para enfrentar estreses hídricos y nutricionales. En el caso específico de los viveros, tiene una mayor capacidad de adaptación de las posturas en el trasplante, porque se obtienen posturas más vigorosas, por lo tanto, es importante estudiar la causa de la baja calidad de las posturas en el sistema productivo cafetalero de Limonar, por lo tanto el objetivo de esta investigación fue evaluar el comportamiento de las posturas de C. arabica L. con dos cepas de hongos micorrízicos arbusculares en el sistema productivo cafetalero de Limonar.

\section{MATERIALES Y MÉTODOS}

El experimento se realizó en el macizo montañoso, ubicado en el Centro Docente Científico Productivo Limonar de la Facultad Agroforestal, perteneciente al municipio El Salvador, provincia Guantánamo, en los meses de septiembre a mayo de 2014, teniendo una extensión de 0,05 hectáreas a una altura de $410 \mathrm{msnm}$.

Característica climática. Las precipitaciones en la zona, van desde los 1114,0 hasta los 1908,3 mm anuales, destacándose los meses de mayo, julio, agosto, septiembre y noviembre con mayores precipitaciones, las temperaturas promedio mínimas de $18,4{ }^{\circ} \mathrm{C}$ y máximas de $29.9^{\circ} \mathrm{C}$. La humedad relativa entre 75 y $86 \%$ y la evaporación promedio anual de 154,16 $\mathrm{mm}$.

Características generales del suelo. El Suelo se clasificó como pardo Sialítico Mullido Carbonatado según Hernández et al. (1999). Las características del suelo donde se condujo el experimento, tuvo valores medios de materia orgánica (MO), de fósforo (P) y de potasio (K). El pH fue ligeramente ácido, y se ubicó dentro de los rangos adecuados para el cultivo del cafeto.

Diseño experimental. Se utilizó un Diseño Completamente Aleatorizado con tres tratamientos y cuatro réplicas. Los tratamientos correspondieron a la inoculación de las semillas de cafeto con dos cepas de hongos micorrízicos arbusculares G. cubense y $R$. intraradices y un tratamiento sin inoculación (control). Se sembraron 360 plantas en bolsa de polietileno, de ellas se tomaron 120para ser evaluadas.

Obtención de los microorganismos empleados. Como inoculantes micorrízicos, se utilizaron las cepas G. cubense y R.intraradices, procedentes de la colección de hongos micorrízicos arbusculares (HMA) del Departamento de Biofertilizantes y Nutrición de las Plantas, del Instituto Nacional de Ciencias Agrícolas (INCA). Se aplicaron en el momento de la siembra, por el método de recubrimiento de las semillas utilizando una dosis de 5,95 kg.ha-1 de EcoMic ${ }^{\circledR}$, equivalente al 10\% del peso de las semillas (Fernández et al., 2000). Para el caso del estiércol vacuno se utilizó la relación 5:1 según las normas técnicas para el cultivo en estas condiciones. 
Evaluaciones. Se escogieron 10 plantas al azar en cada parcela útil, y en ellas se evaluaron las siguientes variables:

Número de hojas/planta. A los 117 días después de realizada la siembra se realizó la cuantificación de las hojas de cada planta individualmente por tratamiento y se utilizó el valor promedio.

Área Foliar $\left(\mathrm{cm}^{2}\right)$. A los 117 días después de realizada la siembra se realizó ante del trasplante para el campo, el largo (L) por el ancho (A) de la hoja multiplicado por el coeficiente según variedad.

$$
\mathrm{AF}=\mathrm{L} \times \mathrm{A} \times 0,64 \text { (C. arabica) }
$$

Altura de las plantas (cm). A los 117 días después de realizada la siembra, haciendo uso de una regla graduada, midiéndose desde el cuello de la raíz de las plantas hasta el ápice.

Largo de la raíz (cm). A los 117 días después de realizada la siembra, haciendo uso de una regla graduada, midiéndose desde el cuello de la raíz de las plantas hasta la cofia.

Porcentaje de materia seca (\%). Las plantas seleccionadas fueron sometidas a una temperatura de $60 \pm 5^{\circ} \mathrm{C}$, hasta lograr masa constante y posteriormente se determinó su porcentaje de masa seca utilizando la fórmula:

Incrementos netos. Se utilizó un índice de eficiencia (IE), reportado inicialmente por Siqueira y Franco (1988) para evaluar la dependencia micorrízica.

Análisis estadístico. Para las variables morfológicas se utilizó un Análisis de Varianza de clasifi- cación simple. Para las diferencias significativas entre las medias se realizó la Prueba de Rango Múltiple de Duncan para $p \leq 0,05$ de probabilidad del error. Para el análisis estadístico fue utilizado el paquete STATGRAPHICS Versión 5.1 en ambiente Windows.

Análisis económico. La valoración económica de los resultados de cada uno de los experimentos, se realizó según la metodología descrita por Riera y Medina, (2005).

Costo $(\$ / L)$. Gastos incurridos por la aplicación del HMA empleado.

Beneficio (\$/ha). Ganancia neta obtenida por diferencia entre el valor de la producción y los costos.

Relación B/C. Cociente obtenido de dividir el beneficio entre el costo total incurrido en las diferentes actividades en cada tratamiento.

Valores de la relación B/C. Mayores a 1, indican el aporte de ganancia y un valor de 2 la obtención de un beneficio del 100\%. Valores de 3 o superiores corresponden a ganancias muy notables.

\section{RESULTADOS Y DISCUSIÓN}

En la Tabla 1, se observa la altura de las plantas a los 117 días mediante un análisis de ANDEVA, el cual demostró diferencia entre los tratamientos inoculados y el control $(\mathrm{P}<0,05)$, donde la inoculación de ambas cepas G. cubense y $R$. intraradices, produjeron efectos muy positivos sobre la altura de las plantas, destacándose en este sentido, última cepa $R$. intraradices. 
Estos resultados, coinciden con Fernández (1999) quien encontró resultados positivos en el incremento de la altura de las posturas de $C$. arabica L. utilizando la cepa de HMA G. cubense debido a las acciones de las hifas y micelio de estos microorganismos que pueden provocar el aumento en las absorciones de nutrimentos que son esenciales para el desarrollo de las plantas con es el caso del fósforo.

Sánchez et al. (2009) encontraron, respuestas positivas en el crecimiento de las posturas de cafeto en los tratamientos inoculados con diferentes cepas de hongos micorrízicos arbusculares respecto a los tratamientos no inoculados, debido que en el suelo se encontraba el suministro de nutrientes necesario para una micorrización efectiva de las posturas de café.

Por otra parte, Ávila et al. (2010) en sus estudios, relacionados con la respuesta del café al fósforo y abonos orgánicos en la etapa de almácigo, informaron que las diferentes proporciones de abonos orgánicos propiciaron el aumento en algunas de las variables evaluadas en el cultivo, infiriendo que los resultados obtenidos, pueden estar dados por la actividad y población de microorganismos que contribuyen en la disponibilidad de nutrimentos para las plantas. Información que nos permite en nuestro trabajo, relacionar los beneficios de la aplicación del estiércol vacuno como fuente de materia orgánica y los efectos positivos de los hongos micorrízicos arbusculares en el desarrollo del cultivo del café.

De manera general, se observó que la inoculación de $R$. intraradices, estimula el desarrollo vegetativo de las posturas, al obtenerse plantas de mayor altura y más vigorosas que en la forma tradicional. Se puede inferir que eso puede resultar de la capacidad de esta cepa en realizar una simbiosis efectiva en éstos tipos de suelo para éste cultivo, dada por la presencia de micelio externo efectivo, capaz de explorar de modo eficiente nutrientes del sustrato transfiriéndolo para la planta.

$\mathrm{Al}$ analizar el efecto de la coinoculación HMA sobre las posturas de café en el área foliar a los 117 días después de la siembra (Tabla 1), se observó que el tratamiento que se inoculó con $R$. intraradices fue el que mejor se comportó con diferencias significativas con respecto a los demás tratamientos, presentando valores óptimos para el desarrollo de posturas, los cuales concuerdan con los resultados reportados por Fernández (1999) y Sánchez (2001) y con índices de eficiencia

Tabla 1. Efecto de la inoculación HMA sobre la altura (AP), Area foliar (AF), índice de eficiencia (IEF), largo de raíz (LR), pares de hojas (PH) y masa seca de follaje (Msf) de las posturas de café a los 117 días después de la siembra en la finca de Limonar en el 2014.

\begin{tabular}{lllllll}
\hline \multicolumn{1}{c}{ Tratamiento } & AP $(\mathbf{c m})$ & $\mathbf{A F}\left(\mathbf{c m}^{2}\right)$ & $\mathbf{I E}(\mathbf{\%})$ & $\begin{array}{c}\text { LR } \\
(\mathbf{c m})\end{array}$ & PH (u) & Msf (g) \\
\hline R. intraradices & $23,0 \mathrm{a}$ & $392,27 \mathrm{a}$ & 37 & $36,0 \mathrm{a}$ & $7,85 \mathrm{a}$ & $3,03 \mathrm{a}$ \\
G. cúbense & $21,0 \mathrm{~b}$ & $351,84 \mathrm{~b}$ & 36 & $30,6 \mathrm{ab}$ & $6,70 \mathrm{~b}$ & $2,94 \mathrm{~b}$ \\
Control & $19,9 \mathrm{c}$ & $257,11 \mathrm{c}$ & & $26,4 \mathrm{c}$ & $5,92 \mathrm{~b}$ & $2,25 \mathrm{c}$ \\
\hline ES $\bar{x}$ & $\mathbf{0 , 2 6 1 2}$ & $\mathbf{1 , 0 1 4}$ & & $\mathbf{0 , 9 3 9}$ & $\mathbf{0 , 2 2 5}$ & $\mathbf{0 , 0 9 3 7}$ \\
\hline
\end{tabular}

Letras iguales no tienen diferencias significativas (Duncan; $\mathrm{p} \leq 0,05$ ). 
de $36-37 \%$ que indica un efecto importante de la micorrización sobre el crecimiento.

Resultados similares fueron reportados por Rivera et al. (2010), quienes encontraron incrementos significativos en el área foliar de las posturas de café en los tratamientos con G. fasciculatum y humus de lombriz, indicando una micorrización efectiva de las posturas de cafetos en éstos suelos.

Los mismos autores, plantean que con estas alternativas se puede disminuir en gran medida el suministro de nutrientes minerales y de ésta manera, impactar en los costos económicos, ecológicos y ambientales, debido a los efectos beneficiosos que presentan los hongos micorrízicos arbusculares en el desarrollo y crecimiento de las plantas.

Ricci et al. (2006) indican que el balance de las proporciones de los abonos orgánicos para el suministro de nutrientes en las posturas de café, resultan ser menores cuando se emplea el uso combinado de los hongos micorrízicos arbusculares en la producción de posturas de café. Lo que está relacionado con los resultados obtenidos en esta investigación, cuando observamos que existe mejor comportamiento en los tratamientos inoculados con HMA al respecto del tratamiento control.

Sadeghian (2008) informó que en Colombia se recomienda la aplicación de 120 - $300 \mathrm{~kg} \cdot \mathrm{ha}^{-1} \cdot \mathrm{año}^{-1}$ de $\mathrm{Na}$ C. arabica de acuerdo con el contendido de materia orgánica del suelo, el nivel de sombra y la densidad de siembra.

La Tabla 1 muestra diferencias significativas entre los tratamientos. Efectos que son muy positivos sobre el largo de la raíz de las posturas y aunque existen diferencias significativas entre el largo de la raíz de los tratamientos inoculados, la importante diferencia entre los índices de eficiencia demuestra que la efectividad de la micorrización fue positiva cuando se compara con el control que no fue inoculado.

En este sentido, los incrementos significativos en la raíz del tratamiento que se la aplicó $R$. intraradices con respecto a los demás tratamientos fueron mayores, posiblemente dado a que el empleo de esta inoculación aumenta la adsorción de nutrientes en el suelo y por ende un mejor desarrollo radicar.

Resultados similares por Sánchez et al. (2011) fueron alcanzados en producción de posturas de cafetos micorrizadas con la cepa $R$. intraradices, quienes encontraron una respuesta positiva tanto sobre el crecimiento y el funcionamiento micorrízico de las posturas con respecto a los tratamientos no inoculados.

Rivera et al. (2006) refieren que la cepa $R$. intraradices ha funcionado adecuadamente en los suelos pardos con carbonatos, para diversos cultivos, bajo el concepto de que el tipo de suelo, es el criterio fundamental para definir cuál o cuáles son las especies y cepas eficientes para una condición edafoclimática dada. Información que está en correspondencia con los resultados obtenidos en esta investigación, dada que la cepa $R$. intraradices tuvo mejor efecto simbiótico para este tipo de suelo con respecto a la cepa G. cubense en la variable largo de la raíz.

Se puede observar en la Tabla 1 a los 117 días después de la siembra, diferencias entre los tratamientos sobre la variable pares de hojas, donde los resultados alcanzados en el tratamiento de la cepa $R$. intraradices mostró 
diferencias significativas con respecto a los dos tratamientos. a cepa G. cubense no mostró diferencias significativas frente al tratamiento control.

Estos resultados se pueden comparar con lo obtenido por Joao (2002) quien observó en las dos repeticiones un efecto significativo de la inoculación con especies de HMA, donde de forma general los mayores valores se obtuvieron en los tratamientos inoculados y los menores en los no inoculados.

Por otra parte, Sánchez et al. (2011) informaron en estudios realizados sobre la utilización de los HMA y humus de lombriz en posturas de café, que ambas combinaciones de estas alternativas, permitenincrementareldesarrolloylaefectividad micorrízica de las posturas inoculadas, indicativo de una simbiosis micorrízica efectiva de las posturas en estas condiciones.

Relacionado a esto Vélez (2012), informó que el aumento del porcentaje de colonización micorrízica, puede estar ocasionado por las acciones que ejercen los HMA en el incremento y la absorción de nutrientes disponibles para las plantas, criterios que están en correspondencia con los resultados obtenidos en nuestra investigación al observar que los HMA ejercieron un efecto positivo en el desarrollo de las posturas de café.

La Tabla 1 muestra la interacción entre los tratamientos inoculados $R$. intraradices y $G$. cubense además del tratamiento control en la masa seca del follaje a los 117 días después de la siembra, observándose incrementos significativos en los tratamientos inoculados respecto al control. Estos resultados son similares a los encontrados por Joao (2002) en los tratamientos inoculados con cepas efectivas, donde hubo una relación directa entre la eficiencia simbiótica, el área foliar y la producción de masa seca.

En este orden, se ha demostrado que el análisis del crecimiento constituye un elemento importante en estudios de producción de materia seca en plantas cultivadas bajo diferentes prácticas agronómicas (fertilización mineral e inorgánica, riego, entre otras) o sometidas a diferentes condiciones ambientales (Riaño et al., 2004). Relacionado con esto, Soto (2006) informó que la producción de biomasa por una planta constituye una variable importante para caracterizar su productividad, pues refleja el rendimiento biológico del vegetal. Por otra parte, los indicadores fisiológicos evaluados pueden generar incremento en la producción, disminuir las enfermedades así como el impacto ambiental, de manera que esta práctica constituye una opción ecológica para el establecimiento del café a grandes escalas de producción.

Valoración económica. La Tabla 2 muestra la valoración económica en USD a partir de los cálculos realizados de los gastos según el método que se aplicó, basándose en la producción de 378 000,00 posturas. En ese análisis se incluyó los valores que se reportan en la carta tecnológica de vivero sobre los costos de los diferentes materiales y actividades que se relacionan con el montaje de viveros.

Se comparó la variante tecnológica de mejor resultado frente a la norma técnica; en este se destaca el tratamiento con $R$. intraradices que disminuyó significativamente los gastos en \$1543,96 USD para producir una postura de optima calidad. 
Tabla 2. Análisis de la factibilidad económica para producir 378 000,00 posturas de café en USD.

\begin{tabular}{lccccc}
\hline Tratamientos & Posturas & V.P $\$ /$ Post & Costo & Beneficio & Relación B:C \\
\hline R. intraradices & 378000,0 & 4279.25 & 1543,96 & 2735,25 & 2 \\
G. cubense & 378000,0 & 4279.25 & 1679,77 & 2599,43 & 2 \\
Control (Sin Inoculación) & 378000,0 & 4279.25 & 2263,06 & 2016,15 & 1 \\
\hline
\end{tabular}

Con relación a esto, la FAO (2002) informó que valores del B/C de tres o superiores corresponden a ganancias muy notables, debido al alto valor de la producción, obtenida en los tratamientos donde se aplican fertilizaciones minerales e inorgánicas, en comparación con los tratamientos controles.

Pérez (2011) informó que la factibilidad de alternativas nutricionales aplicadas en el cafeto, garantizaran elevadas producciones, que harán que las inversiones realizadas sean rentables dentro del propio año.

Con relación a la factibilidad económica Cadena (2005), informó que los productores cafetaleros colombianos están en constante búsqueda de alternativas para una mayor productividad en la producción cafetera y que permita mayor ganancias en las producciones obtenidas.

\section{CONCLUSIONES}

La alternativa ecológica que mejor resultó en cuanto a la inoculación de los hongos micorrízicos arbusculares, fue la cepa $R$. intraradices en las condiciones de estudio.

La aplicación de la cepa $R$. intraradices se comportó como la alternativa más económica, lográndose un ahorro de 719,10 USD con respecto al control.
El mejor comportamiento en cuanto al desarrollo y crecimiento de las posturas de C. arabica $\mathrm{L}$. se logró con la cepa $R$. intraradices en las condiciones de estudio.

\section{REFERENCIAS}

ÁVILA R., W.E., SADEGHIAN K., S., SÁNCHEZ A., P. M. y CASTRO F., H. E. 2010. Respuesta del café al fósforo y abonos orgánicos en la etapa de almácigo. CENICAFÉ. 61(4):358 - 369.

BUSTAMANTE, C., VIÑALS, R., PÉREZ, A., RODRÍGUEZ, M. y ARAÑO, L. 2010. Fertilización mineral y uso de abono verde en $C$. canephora Pierre ex-Froehner cultivado bajo poda sistemática en los macizos montañosos de la Sierra Maestra y SaguaNipe-Baracoa. Informe Final Proyecto Nacional 07.03.087. Documento Interno. Estación Central de Investigaciones de Café y Cacao. La Habana, 90 p.

CADENA, G. 2005. Desarrollos científicos de CENICAFÉ en la última década. Revista de la Academia Colombiana de Ciencias Exactas, Fisicas y Naturales. 29(110):89 - 99.

FERNÁNDEZ, F. 1999. Manejo de las asociaciones micorrízicas arbusculares sobre la producción de posturas de cafeto (C. arabica L. var. Catuay) en algunos tipos de suelos. Tesis de Grado Doctor en Ciencias Agrícolas. Instituto Nacional de Ciencias Agrícolas. La Habana. 102 p.

FERNÁNDEZ, F., GÓMEZ, R., VANEGAS, L. F., NOVAL, B. M. DE LA. Y MARTÍNEZ, M. A. 2000. Producto inoculante micorrizógeno. Oficina Nacional de Propiedad Industrial. Cuba, Patente no. 22641. 
FOOD AND AGRICULTURE ORGANIZATION OF THE UNITED NATIONS (FAO). 2002. Los fertilizantes y su uso. Una guía de bolsillo para los oficiales de extensión. Cuarta Edición, Organización de las Naciones Unidas para la Agricultura y la Alimentación, Asociación Internacional de la Industria de los Fertilizantes. Roma. 87 p.

HERNÁNDEZ, A., PÉREZ, J., BOSCH., D. y RIVERO, L. 1999. Nueva versión de clasificación genética de los suelos de Cuba. Primera edición, Agencia de Información y Comunicación para la Agricultura, Ministerio de Agricultura, La Habana, 349 p.

HERNÁNDEZ, A., VANTOUR, A., MORALES, M. y FUENTES, E. 2006. Suelos dedicados al cafeto en Cuba, pp. 256 - 267. En: Rivera, R. y Soto, F. (eds.). Primera edición. El cultivo del cafeto en Cuba. Investigaciones y Resultados, Instituto Nacional de Ciencias Agrícolas. La Habana, 500 p.

JOAO, J.P. 2002. Efectividad de la inoculación de cepas de HMA en la producción de posturas de cafeto sobre suelo Ferralítico Rojo compactado y Ferralítico Rojo lixiviado de montaña. Tesis de Grado Magister en Ciencias en Nutrición de las Plantas y Biofertilizantes, Instituto Nacional de Ciencias Agrícolas, La Habana, 85 p.

PÉREZ, A. 2011. Fertilización y requerimientos de nitrógeno para plantaciones de Coffea canephora Pierre ex Froehner var. Robusta cultivada en suelos pardos de la región oriental premontañosa de Cuba. Tesis de Grado Doctor en Ciencias Agrícolas, Instituto Nacional de Ciencias Agrícolas, La Habana.106 p.

RIAÑO, N., ARCILA, J., JARAMILLO, A. y CHAVEZ, B. 2004. Acumulación de materia seca y extracción de nutrimentos por Coffea arabica L. cv. Colombia en tres localidades de la zona cafetera central. Cenicafé. 55(4):265 - 276.

RICCI, M., RODRIGUES, B. y RIBEIRO, J. 2006. Cultivo de Crotalaria spectabilis intercalada ao café
Arábica plantado em diferentes espaçamentos sob manejo orgânico. Seropédica: Embrapa Agrobiologia. Documentos, 208. Ministério da Agricultura, Pecuária e Abastecimento. $21 \mathrm{p}$.

RIERA, M. y MEDINA, N. 2005. Influencia de las micorrizas sobre las poblaciones bacterianas y su efecto sobre los rendimientos en secuencias de cultivos. Cultivos Tropicales. 26(4):21 - 27.

RIVERA, R., FERNÁNDEZ, F., FERNÁNDEZ, K., RUIZ, L., SÁNCHEZ, C. y RIERA, M. 2007. Advances in the management of effective arbuscular mycorrhizal symbiosis in tropical ecosystem, pp. 151-196. En: Chantal, H. y Plenchette, C. Mycorrhizae in Crop Production. Haworth Press Inc. New York, 200 p.

RIVERA, R., RUIZ, L., FERNÁNDEZ, F., SÁNCHEZ, C., RIERA, M., HERNÁNDEZ, A., FERNÁNDEZ, A., FERNÁNDEZ, K. y PLANAS, R. 2006. La simbiosis micorrízica efectiva y el sistema suelo-plantafertilizante. En: Congreso Sociedad Cubana de la Ciencia del Suelo. 6:8 -10. La Habana, Cuba.

RIVERA, R., SÁNCHEZ, C., CABALLERO, D., CUPULL, R., GONZÁLEZ, C. y URQUIAGA, S. 2010. Abonos verdes e inoculación micorrízica de posturas de cafeto sobre Suelos ferralíticos rojos lixiviados. Cultivos Tropicales. 31(3):75 - 81.

SADEGHIAN, K.S. 2008. Fertilidad del suelo y nutrición del café en Colombia: Guía Práctica. Boletín Técnico CENICAFÉ. 32:1 - 43.

SÁNCHEZ, C. 2001. Uso y manejo de los HMA y los abonos verdes en la producción de posturas de cafeto. Tesis de Grado Doctor en Ciencias Agrícolas. Instituto Nacional de Ciencias Agrícolas, La Habana.103 p.

SÁNCHEZ, C., CABALLERO, D., RIVERA, R. y CUPULL, R. 2006. Respuesta de cepas de hongos micorrizógenos (HMA) sobre el desarrollo de posturas de cafeto (Parte I). Suelo pardo gleyzoso. Centro Agrícola. 33(1):33 - 38. 
SÁNCHEZ, C., RIVERA, R., BUSTAMANTES, C., PÉREZ, C., CUPULL, R., GONZÁLEZ, C. y FERRER, M. 2003. Efecto de diferentes fuentes de abonos verdes sobre el desarrollo de las posturas de cafetos en un suelo pardo gleyzoso. Centro Agrícola. 30(4):35 - 39 .

SÁNCHEZ, C., RIVERA, R., CABALLERO, D., CUPULL, R., GONZÁLEZ, C. y URQUIAGA, S. 2011. Abonos verdes e inoculación micorrízica de posturas de cafeto sobre suelos ferralíticos rojos lixiviados. Cultivos Tropicales. 32(3):11 - 17.

SÁNCHEZ, S., CABALLERO, D., CUPULL, R., GONZÁLEZ, C., URQUIAGA, S. y RIVERA, R. 2009. Los abonos verdes y la inoculación micorrízica de plántulas de Coffea arabica sobre suelos cambisoles gléyicos. Cultivos Tropicales. 30(1):25 - 30.

SIQUEIRA, J.O. y FRANCO, A.A. 1988. Biotecnología do solo. Fundamento e perspectivas. Brasilia: MEC - Ministerio de Educação, ABEAS; Larras: ESAL, FAEPE. $236 \mathrm{p}$.

SOTO, F. 2006. Crecimiento y requerimientos ecológicos del cafeto, pp. 67-98. En: Rivera, R. y Soto, F. El Cultivo del cafeto en Cuba. Investigaciones y Resultados. Primera edición. Instituto Cubano del Libro. La Habana, 500 p.

VÉLEZ, F. 2012. Efecto de abonos verdes en la agregación y micorrización en el cultivo de maíz (Zea mays L.) en un suelo de ladera de Palmira (Colombia). Tesis de grado Magíster en Ciencias Agrarias, Línea de Investigación en Suelos, Facultad de Ciencias Agropecuarias, Universidad Nacional de Colombia, Sede Palmira. 96 p. 